\title{
ANALISI DEI PRINCIPALI FATTORI DI RISCHIO PREESISTENTI NEI PAZIENTI CON DIAGNOSI DI COVID-19 AD AMAPÁ, AMAZÔNIA, BRASILE
}

\section{ARTICOLO ORIGINALE}

OLIVEIRA, Arthur Aires de ${ }^{1}$, MOREIRA, Danilo José Silva², PIMENTEL, Jhon Allyson Sena ${ }^{3}$, COSTA, Pedro Henrique de Magalhães ${ }^{4}$, DIAS, Claudio Alberto Gellis de Mattos $^{5}$, ARAÚJO, Maria Helena Mendonça de ${ }^{6}$, OLIVEIRA, Euzébio de ${ }^{7}$, DENDASCK, Carla Viana ${ }^{8}$, SOUZA, Keulle Oliveira da ${ }^{9}$, FECURY, Amanda Alves ${ }^{10}$

\section{OLIVEIRA, Arthur Aires de. Et al. Analisi dei principali fattori di rischio preesistenti} nei pazienti con diagnosi di COVID-19 ad Amapá, Amazônia, Brasile. Revista Científica Multidisciplinar Núcleo do Conhecimento. Anno 06, Ed. 06, Vol. 17, pp. 5672. Giugno 2021. ISSN: 2448-0959, Collegamento di accesso: https://www.nucleodoconhecimento.com.br/salute/rischio-preesistenti, DOI: 10.32749/nucleodoconhecimento.com.br/salute/rischio-preesistenti

\footnotetext{
${ }^{1}$ Studioso di medicina. Università Federale di Amapá (UNIFAP).

${ }^{2}$ Studioso di medicina. Università Federale di Amapá (UNIFAP).

${ }^{3}$ Studioso di medicina. Università Federale di Amapá (UNIFAP).

${ }^{4}$ Studioso di medicina. Università Federale di Amapá (UNIFAP).

${ }^{5}$ Biologo, Dottore di Ricerca in Teoria e Comportamento, Professore e ricercatore del Corso di Laurea in Chimica dell'Istituto di Educazione Di Base, Tecnica e Tecnologica di Amapá (IFAP) e del Corso di Laurea in Formazione Professionale e Tecnologica (PROFEPT IFAP).

${ }^{6}$ Dottore, Master in Scienze dell'Insegnamento e della Salute, Professore e ricercatore del Corso di Medicina del Campus Macapá, Università Federale di Amapá (UNIFAP).

${ }^{7}$ Biologo, PhD in Malattie Tropicali, Professore e ricercatore del Corso di Educazione Fisica, Università Federale di Pará (UFPA).

${ }^{8}$ Teologo, PhD in Psicoanalisi Clinica. Si occupa da 15 anni di Metodologia scientifica (Metodo di ricerca) nell'orientamento alla produzione scientifica per studenti di master e dottorandi. Specialista in Ricerche di Mercato e Ricerche Sanitarie Dottorando in Comunicazione e Semiotica (PUC SP).

${ }^{9}$ Scienziato sociale, specialista in gestione e insegnamento dell'istruzione superiore, studente di master in studi antropici in Amazzonia (UFPA).

${ }^{10}$ Biomedicale, PhD in Malattie Tropicali, Professore e ricercatore del Corso di Medicina del Campus Macapá, Università Federale di Amapá (UNIFAP), Prorettore di Ricerca e Studi Universitari (PROPESPG) dell'Università Federale di Amapá (UNIFAP).
} 


\section{RIEPILOGO}

Covid-19 (Coronavirus Disease 2019) è un'infezione causata dal virus SARS-CoV-2, con i primi casi segnalati a dicembre 2019 a Wuhan City. È stato scoperto che nei pazienti con comorbilità, c'è un rischio più elevato di complicanze e mortalità in caso di infezione. La malattia è stata considerata dall'Organizzazione Mondiale della Sanità nel 2020 come un'emergenza di salute pubblica e, in Brasile, il Ministero della Salute ha implementato misure per il monitoraggio epidemiologico dei casi registrati nelle diverse unità federative del paese. Tra questi, Amapá assume alti livelli di incidenza e mortalità. Questo studio mira ad analizzare i principali fattori di rischio precedenti riscontrati nei pazienti con COVID-19 nello stato di Amapá. Per questo, i dati secondari disponibili nel Pannello Coronavirus Amapá sono stati utilizzati attraverso una ricerca condotta il 22 maggio 2020. Sulla piattaforma sono stati raccolti dati sul numero di pazienti a cui è stato diagnosticato covid-19, nonché sul numero di decessi dovuti alla malattia, con valutazione congiunta in ogni argomento di comorbilità riscontrata. Dopo aver raggruppato questi dati in fogli di calcolo di Microsoft Excel, è stata eseguita un'analisi quantitativa e descrittiva di questi dati. Nello Stato, un totale di 28.927 pazienti con diagnosi di COVID-19 sono stati registrati fino al giorno della raccolta. Circa l'1,31\% (378 casi) di questi aveva un fattore di rischio che poteva suscettibili loro di sviluppare complicazioni derivanti dal COVID-19, con diabete e malattie cardiache croniche che erano le condizioni con il maggior numero di record. Sempre nello stato di Amapá, sono stati trovati 420 decessi in pazienti con COVID-19. Di questo totale, il 75,71\% (318 casi) aveva precedenti fattori di rischio per un maggiore rischio di complicanze dovute al COVID-19, con ipertensione e diabete come condizioni predominanti. Con lo studio, è stato notato ad Amapá che gran parte della popolazione di pazienti con diagnosi di malattia e che sono morti aveva una storia di fattore di rischio per COVID-19. A causa della notifica dei fattori di rischio nella situazione di morte che non sono stati registrati nel numero di pazienti diagnosticati, nonché della non specificità inerente alle condizioni dei pazienti monitorati dai meccanismi sanitari dello stato, non è stato possibile eseguire un'analisi più dettagliata sul grado di complicanza e tasso di letalità generato da un dato fattore di rischio e sulla sua associazione con l'infezione da coronavirus. 
Parole chiave: COVID-19, Fattori di rischio, Comorbilità.

\section{INTRODUZIONE}

Nella prima metà di dicembre 2019, il primo caso di polmonite di origine sconosciuta era stato segnalato a Wuhan, nella provincia di Hubei, in Cina. Successivamente, un focolaio di polmonite da sindrome respiratoria acuta grave (SRAG) ha afflitto la provincia, attirando l'attenzione dell'Organizzazione Mondiale della Sanità (OMS), che, a partire dalla circoscrizione delle sue attribuzioni, ha dichiarato che la situazione era un'epidemia causata da un nuovo sottotipo di virus appartenente alla famiglia coronaviridae, coronavirus-2, costituendo un'emergenza di salute pubblica di importanza internazionale (ESPII), il 30 gennaio 2020 (COSTA et al., 2020a; FERRARI, 2020; GAUTRET et al., 2020; GUAN et al., 2020; LI et al., 2020; LIMA et al., 2020; MARTINS-CHAVES; GOMES; GOMEZ, 2020; OLIVEIRA et al., 2020; RENU; PRASANNA; GOPALAKRISHNAN, 2020; RODRÍGUEZ-COLA et al., 2020; TAY et al., 2020; ZHAl et al., 2020).

Nel febbraio 2020, il direttore generale dell'OMS ha nominato la malattia coronavirus19 (COVID-19), un'infezione causata da SARS-CoV-2 (dell'espressione in inglese: Severe Acute Respiratory Syndrome Coronavirus 2), uno dei sette sottotipi noti di coronavirus (COSTA et al., 2020a; RENU; PRASANNA; GOPALAKRISHNAN, 2020; HONG et al., 2020).

Dal punto di vista brasiliano, l'infezione da COVID-19 è stata dichiarata dal Ministero della Salute (MS) come emergenza di salute pubblica di importanza nazionale (ESPIN) il 3 febbraio 2020 e ha avuto il suo primo caso confermato in Brasile il 26 febbraio (OLIVEIRA et al., 2020; OLIVEIRA; LUCAS; IQUIAPAZA, 2020). Come misura di supporto al Sistema Sanitario, il Dipartimento di Informatica del Sistema Sanitario Unificato (DATASUS), attraverso il Piano di Emergenza, ha agito fornendo assistenza virtuale alla popolazione attraverso l'applicazione "Coronavírus SUS" (BRASIL, 2020a). 
In Brasile, l'andamento della pandemia è monitorato principalmente dalla MS attraverso indici statistici che riflettono lo stato di ciascuna unità federativa (UF), e lo Stato di Amapá assume elevati indici epidemiologici, in particolare per quanto riguarda il coefficiente di incidenza del COVID-19 (BRASIL, 2020c).

Fino al 17 luglio, 2020, Amapá ha guidato il Nord brasiliano nella classifica del coefficiente di incidenza COVID-19 per UF di notifica (numero di casi confermati ogni 100.000 abitanti), il cui valore era di $3.637,4$ seguito da Roraima, con $3.327,1$, che a sua volta è stato sottosequenzato dallo Stato di Amazonas, con 1962,0. Per quanto riguarda il coefficiente di mortalità COVID-19 (numero di decessi ogni 100.000 abitanti), Amapá era al $7^{\circ}$ posto $(55,2)$ (BRASIL, 2020c).

I fattori di rischio si riferiscono a qualsiasi condizione che comprometta la risposta immunitaria del corpo a una malattia. Pertanto, questi fattori includono sia comorbilità, cioè malattie croniche precedentemente installate e che agiscono concomitante nell'organismo del paziente, sia particolarità degli individui, vale a dire: condizione puerperale, fumo, consumo di alcol, tra gli altri (PIRES; CARVALHO; XAVIER, 2020; ENGIN; ENGIN; ENGIN, 2020; KHALIQ; PHOSWA, 2020).

Gli studi suggeriscono che i pazienti infetti da COVID-19 che hanno comorbilità hanno una maggiore vulnerabilità immunologica e un tasso di letalità più elevato rispetto ai pazienti affetti solo dal virus. Le principali comorbilità elencate includono ipertensione, diabete, malattie cardiache e malattie croniche dell'apparato respiratorio (COSTA et al., 2020a; FERRARI, 2020; MARTINS-CHAVES; GOMES; GOMEZ, 2020; RENU; PRASANNA; GOPALAKRISHNAN, 2020; YANG et al., 2020).

Dato il panorama globale e in considerazione dello stato di Amapá, è essenziale effettuare analisi, studi e promozioni nella ricerca scientifica, al fine di stabilire adeguate gestione per i pazienti affetti da malattie sottostanti e/o condizioni che rappresentino potenziali fattori di rischio per la prognosi dei pazienti con diagnosi di SARS-CoV-2, con l'obiettivo di mitigare le complicanze da essi presentate (RENU; La commissione per i trasporti e il 2000 ha GOPALAKRISHNAN, 2020; YANG e altri, 2020). 


\section{OBIETTIVI}

Analizzare la distribuzione dei principali fattori di rischio preesistenti nei pazienti a cui è stato diagnosticato covid-19 nello stato di Amapá, Amazon, Brasile.

\section{METODOLOGIA}

Questo studio mirava a fare un'analisi quantitativa e descrittiva del numero di pazienti affetti da COVID-19 ad Amapá che attestano fattori di rischio per la malattia, e per questo, è stata effettuata una consultazione il 22 maggio 2020 presso il Coronavirus Amapá Panel (painel.corona.ap.gov.br).

Nella pagina principale del Coronavirus Amapá Panel, è stato cliccato su "download CSV" per ottenere i dati principali dei casi di covid-19 nello stato di Amapá. Quindi, nelle "Comorbilità dei pazienti confermati" della stessa pagina, è stata selezionata l'opzione "Ispeziona" nell'angolo in alto a destra per generare un elenco di comorbilità e la loro relazione numerica. Lo stesso processo è stato ripetuto nelle "Comorbidities of Dying Patients". Successivamente, per ciascuno degli elenchi ottenuti, è stata selezionata l'opzione "CSV daatted" nel campo "Scarica CSV" per ottenere i file e utilizzarli in Microsoft Excel.

All'interno di questi file, alcune comorbilità e situazioni di rischio sono state unite nello stesso set al fine di standardizzare i risultati. Essere: sono raggruppati -in Malattie cardiache croniche- malattie cardiache croniche, malattie cardiache croniche, malattie cardiache croniche, malattie cardiache croniche, malattie cardiovascolari, malattie cardiache, malattie cardiopatiche, malattie cardiache croniche, malattie cardiache; sono anche in - Malattie respiratorie croniche - malattie respiratorie croniche, malattie respiratorie croniche, malattie respiratorie croniche scompensate; Sono ancora in Ipertensione- ipertensione, HAS, ipertensivo; di nuovo sono in - Malattie cardiache e ipertensione- Malattie cardiache croniche (HAS), malattie cardiache croniche - HAS, malattie cardiovascolari tra cui ipertensione, malattie cardiache croniche HAS, malattie cardiache (HAS), malattie cardiache croniche - HAS; così come in Immunosoppressione- immunosoppressione, portatore di malattie cromosomiche o 
stato FR; Ancora una volta ci sono in - Malattie cardiache e diabete - malattie cardiache croniche e diabete, cuore e diabetico; Quindi sono in gravidanza ad alto rischio - donna incinta ad alto rischio, donna incinta ad alto rischio; Infine sono in Malattie renali croniche in fase avanzata - Malattia renale cronica in fase avanzata, malattia renale cronica a stadio avanzato, malattia renale cronica in fase avanzata.

Nel foglio di calcolo "Comorbilità dei pazienti in morte", il numero di casi registrati come "Nessuna informazione" è stato ignorato a causa dell'inconcludenza di questo taglio. Gli altri dati sono stati selezionati e raggruppati come descritto sopra.

Il presente studio ha utilizzato i dati secondari disponibili nel settore dell'accesso del pubblico e ha tenuto conto degli aspetti etici basati sulla Risoluzione 466/12.

\section{RISULTATI}

Tra i 28.927 casi confermati di COVID-19 ad Amapá che sono stati segnalati fino al giorno della raccolta, circa l'1,31\% dei pazienti (378 casi) aveva un fattore di rischio che poteva suscettibili loro di sviluppare complicazioni dovute al coinvolgimento della SARS-CoV-2, come mostrato nella figura 1. 
Figura 1 - Presenza di fattori di rischio nei pazienti con diagnosi di COVID-19 ad Amapá fino al 22 maggio 2020.

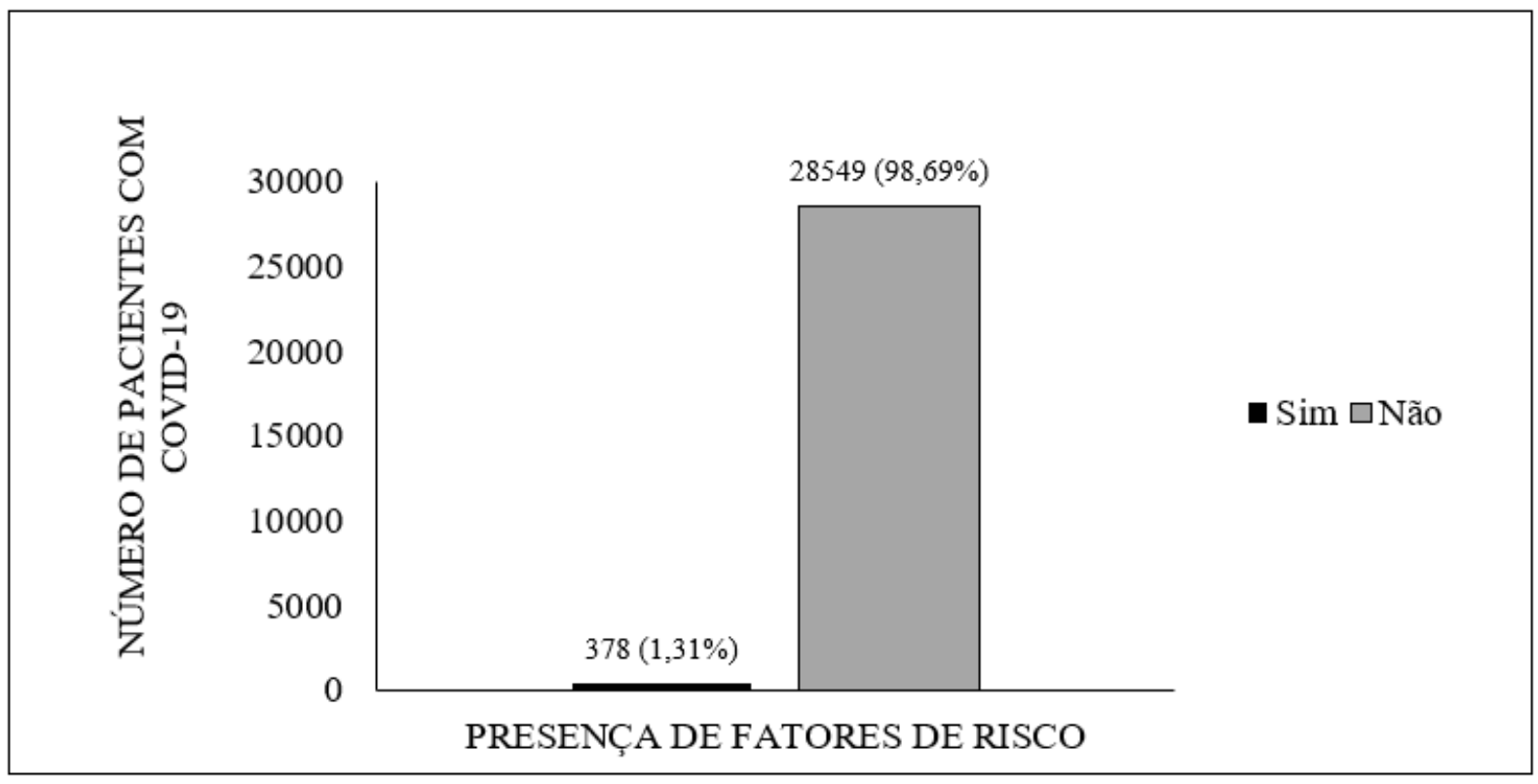

Fonte: Pannello Coronavirus Amapá.

Per quanto riguarda i pazienti che avevano fattori di rischio, il 5,02\% di loro (19 casi) ne aveva più di uno. Le associazioni includono malattie cardiache e ipertensione $(3,44 \%)$, malattie cardiache e diabete $(1,06 \%)$, angioplastica e ateromatosi coronarica $(0,26 \%)$ e malattie renali croniche e diabete $(0,26 \%)$.

Per quanto riguarda i principali fattori di rischio segnalati da soli dai pazienti affetti, si è riscontrato che la maggior parte aveva il diabete del 29,89\% (112 casi), seguito da malattie cardiache croniche con il $28,84 \%$ (109 casi).

Si nota che le malattie della sfera cardiologica sono presenti in modo maggioritario, considerando che 173 pazienti in totale sono affetti da malattie di questa classe, aggiungendo individui con molteplici fattori di rischio e individui solo con malattie cardiache. 
Tabella 1 - Distribuzione dei fattori di rischio nei pazienti con diagnosi di COVID-19 ad Amapá.

\begin{tabular}{|c|c|c|}
\hline Fattori di rischio & $\begin{array}{l}\text { Numero di } \\
\text { relazioni }\end{array}$ & $\begin{array}{l}\text { Percentuale } \\
\text { (\%) }\end{array}$ \\
\hline Diabete & 113 & $29,89 \%$ \\
\hline Malattie cardiache croniche & 109 & $28,84 \%$ \\
\hline Malattie respiratorie croniche & 71 & $18,78 \%$ \\
\hline ipertensione & 46 & $12,17 \%$ \\
\hline Malattie cardiache e ipertensione & 13 & $3,44 \%$ \\
\hline immunosoppressione & 11 & $2,91 \%$ \\
\hline Malattie cardiache e diabete & 4 & $1,06 \%$ \\
\hline Gravidanza ad alto rischio & 4 & $1,06 \%$ \\
\hline $\begin{array}{l}\text { Malattia cronica cronica a stadio } \\
\text { avanzato }\end{array}$ & 3 & $0,79 \%$ \\
\hline Angioplastica e ateromatosi coronarica & 1 & $0,26 \%$ \\
\hline Malattie cardiache croniche e diabete & 1 & $0,26 \%$ \\
\hline Cirrosi epatica & 1 & $0,26 \%$ \\
\hline depressione & 1 & $0,26 \%$ \\
\hline Totale & 378 & $100,00 \%$ \\
\hline
\end{tabular}

Fonte: Pannello Coronavirus Amapá.

In totale, al momento dello studio sono stati registrati 420 decessi. Di questo quantitativo, è stato scoperto che 318 pazienti avevano una storia di comorbilità $o$ condizione predisponente alle complicanze. 
Figura 2 - Presenza di fattori di rischio nei pazienti morti con COVID-19 ad Amapá fino al 22 maggio 2020.

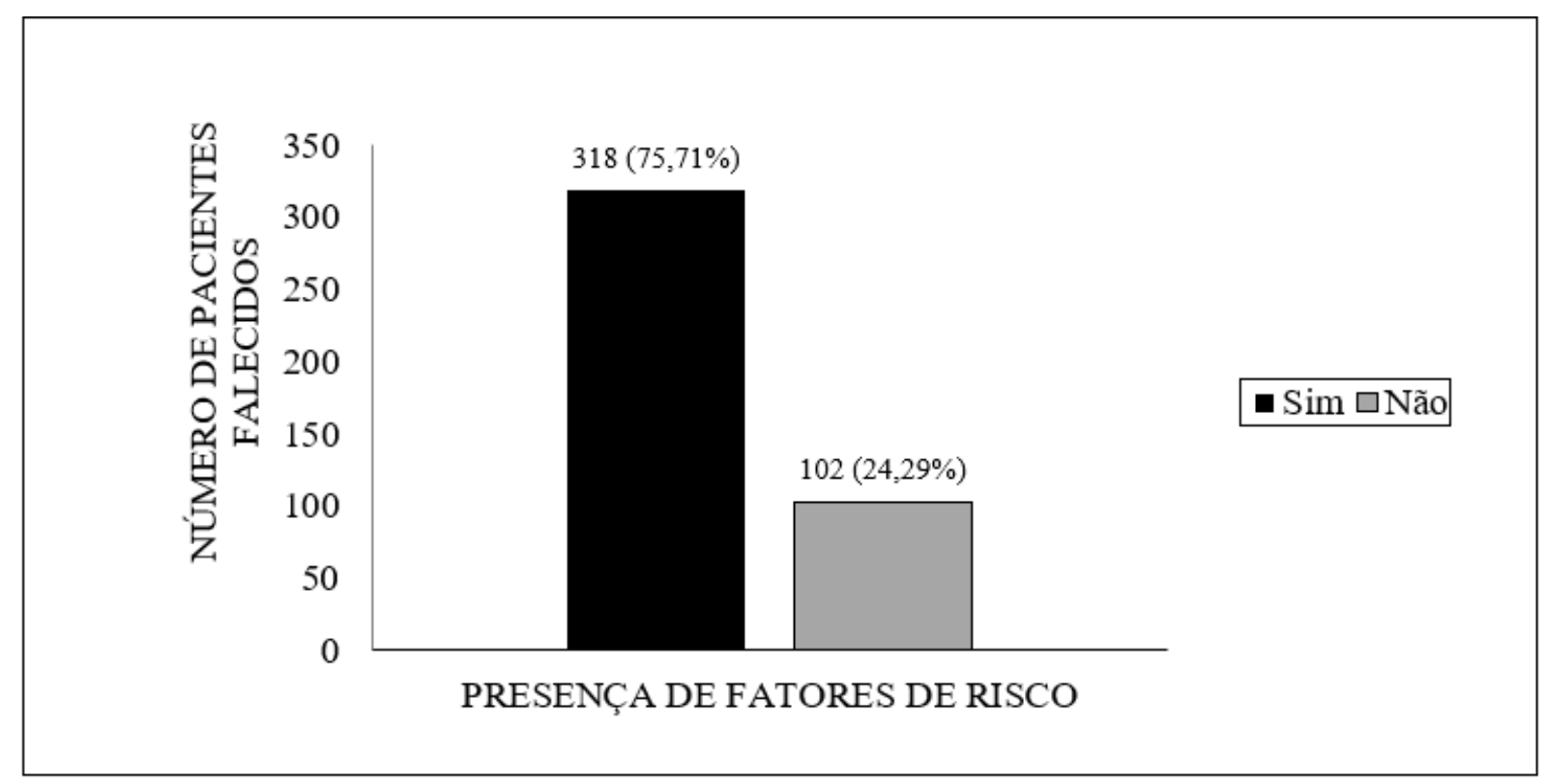

Fonte: Pannello Coronavirus Amapá.

Per quanto riguarda i fattori di rischio presenti nei pazienti deceduti, il record principale è stata l'ipertensione, presente nel $38,68 \%$ dei pazienti (123 casi). È stata anche notata la presenza di altri fattori aggravanti che non erano descritti in precedenza in individui con infezione confermata. Sono obesità, malattia cerebrovascolare, neoplasia, fumo, Alzheimer, malattia del pancreas, dengue, consumo di alcol e periodo puerperale. 
Tabella 2 - Distribuzione dei fattori di rischio registrati nei pazienti deceduti con COVID19 ad Amapá.

\begin{tabular}{|l|c|c|}
\hline Comorbilità & Numero di decessi & Percentuale (\%) \\
\hline ipertensione & 123 & $38,68 \%$ \\
\hline Diabete & 93 & $29,25 \%$ \\
\hline Malattie della pelle & 27 & $8,49 \%$ \\
\hline cuore & 19 & $5,97 \%$ \\
\hline obesità & 19 & $5,97 \%$ \\
\hline Malattie respiratorie & 15 & $4,72 \%$ \\
\hline Malattie cerebrovascolari & 8 & $2,52 \%$ \\
\hline Malattie immunosoppressive & 3 & $0,94 \%$ \\
\hline Neoplasie & 3 & $0,94 \%$ \\
\hline affumicatura & 2 & $0,63 \%$ \\
\hline Alzheimer & 1 & $0,31 \%$ \\
\hline Malattia del pancreas & 1 & $0,31 \%$ \\
\hline Malattia del fegato & 1 & $0,31 \%$ \\
\hline dengue & 1 & $0,31 \%$ \\
\hline Etilista & 1 & $0,31 \%$ \\
\hline Puerpera & 1 & $0,31 \%$ \\
\hline Totale & $\mathbf{3 1 8}$ & $\mathbf{1 0 0 , 0 0 \%}$ \\
\hline
\end{tabular}

Fonte: Painel Coronavírus Amapá.

\section{DISCUSSIONE}

Gli stati dell'Amazzonia del Brasile hanno notevoli discrepanze socioeconomiche rispetto agli altri, una situazione che può riflettere il maggiore impatto sul sistema sanitario osservato. Nello Stato di Amapá, dopo che il primo caso è stato segnalato il 25 marzo 2020, il COVID-19 si è diffuso in modo massiccio, così come nelle altre unità federative (DIAS et al., 2020; MENDONÇA et al., 2020). 
II numero di pazienti con COVID-19 che hanno fattori di rischio per la malattia in Amapá, rispetto al numero totale di pazienti infetti, è considerato basso. C'è consenso in letteratura sul fatto che le comorbilità e le situazioni di vulnerabilità immunologica sono considerate fattori aggravanti per la condizione COVID-19, e questo fatto potrebbe aver spinto gli individui dei gruppi a rischio a raddoppiare le loro cure (COSTA et al., 2020b; RODRÍGUEZ-COLA et al., 2020).

In uno studio condotto da Wang et al. (2020), è stato riferito che dei 36 pazienti che hanno richiesto cure nell'Unità di Terapia Intensiva (UTI), 26 (72,2\%) aveva comorbilità, una scoperta che indica che una storia di malattie croniche può portare allo sviluppo di complicanze. Silva et al. (2020), in uno studio di caratterizzazione clinica ed epidemiologica con 1560 pazienti a cui è stato diagnosticato Covid-19 dalla città di Macapá-AP, ha rilevato che 102 pazienti (6,54\%) comorbilità.

Le principali comorbilità registrate da sole nei pazienti nel presente studio sono state diabete, malattie cardiache croniche, malattie respiratorie croniche e ipertensione. Una meta-analisi fatta con un totale di 1576 partecipanti da Yang et al. (2020) evidenzia anche ipertensione $(21,1 \%)$, diabete $(9,7 \%)$, malattie cardiovascolari $(8,4 \%)$ e malattie dell'apparato respiratorio $(1,5 \%)$ come le comorbilità più frequenti. Silva et al. (2020) ha rilevato che dei 102 pazienti con comorbilità diagnosticate con COVID-19 valutate, malattie cardiovascolari croniche $(38,33 \%)$, diabete $(24,16 \%)$ malattie respiratorie croniche $(13,33 \%)$ sono stati i fattori di rischio più diffusi. Queste condizioni sono associate ad un alto rischio di gravi manifestazioni di COVID-19 (GOMES; GOMEZ, 2020; MARTINS-CHAVES), che è spiegato in alcune parti dalla maggiore espressione dell'enzima convertitore di angiotensina 2 (ECA2) nelle cellule epiteliali alveolari, cardiache, $\beta$ pancreatiche e vascolari dell'endotelio. ECA2 consente l'ingresso del virus nelle cellule del corpo, un fatto che conferma il fallimento degli organi corrispondenti durante la condizione infettiva (ANGHEBEM; REGO; PICHETH, 2020; ASKIN; TANRIVERDI; ASKIN, 2020).

Alcuni pazienti avevano anche altre comorbilità e fattori di rischio, tra cui immunosoppressione, gravidanza ad alto rischio, malattie renali croniche, angioplastica con ateromatosi coronarica, cirrosi epatica e depressione. 
I dati del presente studio mostrano una letalità per i pazienti con malattie immunosoppressive del 27,27\%. Si ritiene che più compromessa è l'immunità dei pazienti con COVID-19, peggiore è la prognosi della malattia, una ragione per cui inquadra i pazienti immunosoppressi come gruppi a rischio per la malattia (GOMES; GOMEZ, 2020; MARTINS-CHAVES). Tuttavia, c'è un rapporto in letteratura che la maggior parte dei pazienti immunosoppressi valutati ha seguito con una buona prognosi. Uno studio condotto da Hrusak et al. (2020) con pazienti pediatrici sottoposti a trattamento antitumorali ha mostrato che su 9 bambini a cui è stato diagnosticato covid-19, 8 sono seguiti da sintomi lievi o addirittura asintomatici. E poi Bussalino et al. (2020) affermano che il mantenimento dell'immunosoppressione può rendere lieve il decorso del COVID-19 per il paziente riducendo la tempesta di citochine caratteristica della malattia.

Per quanto riguarda la gravidanza, nello Stato sono stati segnalati 4 casi di gravidanza ad alto rischio. I rischi materni sono sospetti perché nel corpo della madre vengono innescati cambiamenti immunologici sistemici e locali, che vanno da uno stato proinfiammatorio a uno stato antinfiammatorio (LIU et al., 2020). Nel caso di donne incinte con COVID-19, una meta-analisi fatta da Trippela et al. (2020) con 275 donne incinte colpite dalla malattia hanno dimostrato che 269 hanno descritto la loro sintomatologia e il $91,82 \%$ ha avuto sintomi, tra cui febbre e tosse come i più comuni, e l'8,18\% asintomatico. La maggior parte dei pazienti presenta sintomi lievi o asintomatici, con pochi rapporti sullo sviluppo di complicanze (CAPARROS-GONZALEZ, 2020; TRIPPELA et al., 2020).

In relazione alle malattie coronarie croniche, solo 3 casi confermati sono stati segnalati al sistema di sorveglianza epidemiologica del coronavirus Amapá. Tuttavia, nel sistema sono stati inseriti 27 casi di decessi di pazienti che in precedenza avevano comorbilità di questa classe di malattie, suggerendo una possibile sottosegnalazione delle comorbilità. In uno studio di coorte prospettico prodotto da Cheng et al. (2020) con 701 individui affetti da COVID-19, si è notato che il $26,7 \%$ delle persone analizzate aveva ematuria e il 43,9\% proteinuria. Questi risultati clinici suggeriscono la presenza di problemi di laboratorio (MOITINHO et al., 2020). Dato che il SARS-CoV-2 agisce 
direttamente sul ricevitore ECA2 (ASKIN; Tanriverdi, Presidente in prima linea, signor Presidente in una nota ASKIN, 2020) e che ha già evidenziato una sovraespressione di questo enzima nelle cellule tubolari prossimali di pazienti con malattie orali croniche (FAN et al., 2020), si ritiene che la storia delle malattie della vita possa aggravare il quadro del COVID-19 (RENU; PRASANNA; GOPALAKRISHNAN, 2020). Inoltre, è stato associato alla preesistenza delle malattie renali con la compromissione della funzionalità del sistema immunitario del paziente, un processo che potrebbe essere spiegato dal processo infiammatorio stabilito durante la nefropatia. Ciò promuove l'idea che queste malattie siano fattori di rischio per COVID-19 (OYELADE; ALQAHTANI; CANCIANI, 2020).

Per quanto riguarda la relazione tra angioplastica e ateromatosi coronarica con COVID-19, non sono stati trovati rapporti in letteratura che potessero supportare l'associazione tra questi fattori di rischio e l'infezione da virus.

Dei casi segnalati di malattie del fegato nello stato di Amapá, è stato segnalato solo 1 caso di cirrosi epatica. È stata anche registrata la morte di un paziente che aveva precedenti malattie del fegato, ma non è possibile affermare che sia lo stesso paziente poiché la malattia preesistente non è stata specificata. La letteratura mostra che l'infezione da SARS-CoV-2 coinvolge anche il fegato (AGHAGOLI et al., 2020), e la presenza di malattie del fegato è considerata un fattore determinante per la gravità del quadro COVID-19 (RENU; La commissione per i trasporti e il 2000 ha GOPALAKRISHNAN, 2020). Una meta-analisi di Oyelade, Alqahtani e Canciani (2020) che ha coinvolto 5.595 pazienti ha mostrato una prevalenza di malattie del fegato in un paziente con COVID-19 del 3\%, e di questi, la gravità è stata riportata nel 57,33\%. Lo stesso studio ha rivelato un tasso di mortalità del 17,65\%. Nonostante la gravità, non c'era alcuna correlazione nella letteratura tra la malattia epatica preesiste e il risultato del COVID-19.

Nell'unico caso di depressione registrato nello Stato, c'è una preoccupazione della comunità scientifica circa la possibilità che le malattie neuropsichiche costituiscano anche un rischio per la condizione COVID-19. I disturbi della salute mentale, così come lo stato di disagio psicologico, influenzano direttamente il funzionamento del sistema 
immunitario di un individuo, che può suscettibili alle infezioni virali delle vie respiratorie, che evidenzia una possibile relazione tra lo stato emotivo del paziente e il grado di coinvolgimento da parte della SARS-CoV-2 (RAJKUMAR, 2020). Tuttavia, gli effetti generati dal virus sui pazienti in questione, così come la risposta immunologica generata dall'organismo, non sono ben chiariti in letteratura (TROYER; KOHN; HONG, 2020). Per i pazienti con una storia di debolezza della salute mentale, si dovrebbe prestare maggiore attenzione alla condizione, poiché può ricaduta o peggiorare in mezzo allo scenario pandemico installato e a una possibile diagnosi di COVID-19 (YAO; CHEN; XU, 2020).

I dati raccolti dal pannello coronavirus di Amapá indicano che il 76\% dei pazienti morti aveva comorbilità o fattori aggravanti preesiste. Un'indagine epidemiologica di Almeida et al. (2020) nello Stato di Maranhão ha scoperto che dei 100 pazienti a cui è stato diagnosticato covid-19 e che sono morti, il 78\% aveva comorbilità precedenti, assumendo un modello simile a quello osservato ad Amapá.

Si osserva che l'ipertensione è il fattore di rischio più registrato tra i pazienti con amapaense morti. Tuttavia, non è possibile determinare il tasso di letalità per i pazienti ipertesi, poiché il numero di casi confermati della malattia è inferiore a quello riportato di morte. Tuttavia, la letteratura mostra che questa malattia è uno dei principali fattori aggravanti per la condizione COVID-19, insieme ad altre malattie cardiovascolari (AGHAGOLI et al., 2020; ALMEIDA et al., 2020; FERRARI, 2020). II meccanismo fisiopatico proposto per spiegare questa relazione coinvolge il sistema reintina. $A$ causa del più alto legame tra SARS-CoV-2 e ECA2, la concentrazione di questo enzima è ridotta nell'ospite, con conseguente alti livelli di angiotensina II e diminuzione dell'angiotensina 1-7, effetti che generano vasocostrizione e ritenzione di sodio e peggiorano la condizione ipertensiva (TADIC et al., 2020).

Per quanto riguarda la relazione tra diabete e COVID-19, è possibile peggiorare le condizioni cliniche del paziente, considerando che sars-COV-2 promuove un aumento della glicemia nei pazienti con diabete di tipo 2 (BORNSTEIN et al., 2020). II peggioramento delle condizioni dei diabetici può essere correlato al fatto che questa comorbilità diminuisce l'immunità naturale del corpo (COSTA et al., 2020b; MA; HOLT, 
2020). In relazione ai casi segnalati di questa malattia ad Amapá, è evidente che il numero di pazienti confermati (112) è quantitativamente vicino a coloro che sono morti (93), corroborando così l'idea che il diabete possa aggravare la condizione di covid-19 al punto che il paziente muore (COSTA et al., 2020b; RONCON et al., 2020).

Un'altra caratteristica importante del diabete e della relazione SARS-COV-2 sono i meccanismi di complicanze derivanti dall'unione di questi due fattori, poiché gli individui che hanno il diabete e sono infettati da un virus sono più a rischio di sviluppare complicazioni (LACOBELLIS, 2020). Un'ipotesi sul diabete che agisce negativamente, insieme al virus, è correlata all'ECA2, poiché è presente in diversi tessuti e funge da porta d'accesso agli agenti infettivi sulla superficie cellulare (AGHAGOLI et al., 2020; MA; HOLT, 2020). Inoltre, i pazienti che utilizzano inibitori enzimatici soppressivi dell'angiotensina (IECAs) hanno maggiori probabilità di sviluppare complicazioni a causa della maggiore produzione di ACE2, che favorisce l'azione del SARS-CoV-2 (FANG; KARAKIULAKIS; ROTH, 2020; FERRARI, 2020; MA; HOLT, 2020).

Le malattie cardiache, a loro volta, sono condizioni associate a prognosi peggiori e alti tassi di letalità (AGHAGOLI et al, 2020). Una meta-analisi di Huang, Wang e Li (2020) ha rilevato che dei 41 pazienti con COVID-19 valutati, 6 (15\%) aveva malattie della sfera cardiaca. In effetti, c'è un numero considerevole di pazienti con malattie cardiache che sono morti ad Amapá (19), generando un tasso di letalità del 15,07\%. La letteratura associa questa gravità alla presenza di recettori ECA2 nel cuore, che sono altamente espressi nell'organo, che possono portare allo sviluppo di lesioni miocardiali acute e danni cardiovascolari cronici (AGHAGOLI et al, 2020). Inoltre, l'effetto avanzato di età e immunosenescenza osservato nella maggior parte dei pazienti con malattie cardiache può essere associato alla prognosi della condizione COVID-19 (DRIGGIN et al., 2020).

È stato osservato anche un numero considerevole di pazienti con malattie respiratorie, che corrisponde a un tasso di letalità del $21,12 \%$ tenendo conto del numero di pazienti con diagnosi di COVID-19 che hanno riportato comorbilità preesistenze. La preesistenza di malattie respiratorie è associata a gravi condizioni di COVID-19 (MARTINS-CHAVE; GOMES; GOMEZ, 2020; YANG et al., 2020). È noto che il sistema 
immunitario risponde all'infezione da SARS-CoV-2 con un processo infiammatorio segnato dall'azione delle cellule immunitarie, necrosi tissutale e iperplasia locale, effetti che possono compromettere il processo di scambio di gas dei polmoni e generare un quadro di polmonite grave (GIMENEZ et al., 2020), il che può giustificare il motivo per cui le malattie respiratorie sono un fattore di rischio per COVID-19.

Sempre nelle informazioni fornite dal Coronavirus Amapá Panel, si osserva che alcuni fattori di rischio per COVID-19 riscontrati nei pazienti deceduti non sono stati riportati dalla sessione di fattori di rischio dei pazienti a cui è stata diagnosticata la malattia, come obesità, malattie cerebrovascolari, neoplasie, fumo, morbo di Alzheimer, malattia pancreatica, dengue, alcolismo e periodo puerperale. Ciò impedisce un'analisi affidabile dello scenario epidemiologico della località. Tuttavia, alcuni aspetti di queste condizioni sono stati discussi in letteratura.

A proposito di obesità, è noto da tempo che è direttamente associato a scarse prognosi e recupero dispendioso in termini di tempo nei casi di infezioni virali. Questa scoperta è attribuita al costante stato infiammatorio caratteristico degli individui obesi, supportato da alti livelli di farmaci infiammatori, che contribuisce alla compromissione della risposta immunologica generata da questi pazienti e, di conseguenza, alla diminuzione della capacità di combattere gli agenti infettivi (COSTA et al., 2020b; HUSSAIN et al., 2020; KORAKAS et al., 2020). Nel contesto del COVID-19, altri fattori associati all'obesità possono essere responsabili delle maggiori possibilità di peggioramento del quadro clinico, che include meccanica respiratoria carente, basso tasso di scambio di gas nei polmoni, aumento della resistenza alle vie aeree, ridotta forza polmonare e minore volume polmonare (STEFAN et al., 2020). Una meta-analisi condotta da Hussain et al. (2020) ha mostrato che dei 2.451 pazienti con indice di massa corporea (IMC) superiore a $25 \mathrm{~kg} / \mathrm{m} 2$ analizzati, 531 sono morti, corrispondenti a un tasso di letalità del $21,66 \%$. Lo stesso studio ha anche valutato pazienti con IMC inferiore a $25 \mathrm{~kg}^{/ \mathrm{m} 2}$, per un totale di 24.506 . Di questi, 1701 morirono, un tasso di letalità del $6,94 \%$. Il confronto tra questi valori dimostra che sovrappeso e obesità possono influenzare la prognosi del paziente. 
Le malattie cerebrovascolari sono state segnalate in casi più gravi di COVID-19 (WANG et al., 2020). II meccanismo alla base dell'aumento della gravità per questa classe di pazienti è ancora incerto, anche se è già stato suggerito che sia simile a quello delle malattie cardiovascolari (PRANATA et al., 2020).

Per quanto riguarda la relazione tra neoplasie e COVID-19, si ritiene che i pazienti oncologici siano suscettibili allo sviluppo di complicanze dovute all'indebolimento della loro attività immunologica (ASOKAN; RABADIA; YANG, 2020; AL-QUTEIMAT; AMER, 2020). Infatti, uno studio retrospettivo di Zhang et al. (2020) ha dimostrato che, su 28 pazienti oncologici valutati, 8 sono morti (tasso di mortalità del $28,6 \%$ ), indicando una prognosi infausta per i pazienti con preesistenza di neoplasie.

La storia del fumo a sua volta è ipotizzata come un importante fattore di rischio per lo sviluppo di condizioni gravi della malattia, poiché il fumo è associato a una salute polmonare compromessa (VARDAVAS; NIKITARA, 2020). Sebbene sia stato segnalato un solo caso di fumo nei pazienti morti nella nostra analisi epidemiologica, ci sono studi che dimostrano un aumento del rischio di gravità nei pazienti con una storia di fumo. Alqahtani et al. (2020), in una meta-analisi che ha coinvolto 139 fumatori attuali e 28 ex fumatori a cui è stato diagnosticato covid-19, ha rilevato che 31 fumatori attuali $(22,30 \%)$ e 13 ex fumatori (46\%) peggiorato delle loro condizioni cliniche. Nello stesso studio, sono morti 5 fumatori attuali dei 13 che hanno sviluppato complicazioni (tasso di mortalità del $38,5 \%$ ). Queste osservazioni non fanno che rafforzare il fatto che gli individui che hanno già fumato o fumato dovrebbero evitare la pratica nel bel mezzo della pandemia e adottare sempre misure preventive.

Sul morbo di Alzheimer, l'associazione tra età avanzata e demenza, caratteristiche notevoli della malattia, nonché la possibile compromissione dello sviluppo neurocognitivo di questi individui durante l'isolamento sociale nella pandemia, incoraggiano la comunità scientifica a valutare la pre-esistenza dell'Alzheimer come fattore di rischio per COVID-19 (BRASIL, 2020b; FERINI-STRAMBI; SALSONE, 2020). In caso di mortalità, Bianchetti et al. (2020) ha rilevato che su 82 pazienti a cui è stato diagnosticato COVID-19 e con demenza, 51 (62,2\%) è morto, un tasso superiore a quello del $26,2 \%$ osservato nei pazienti che non avevano demenza, il che 
sottolinea che non solo il morbo di Alzheimer ma anche altre demenze possono essere associate a una grave condizione di COVID-19.

II caso segnalato di un paziente etilista morto suggerisce l'abitudine come possibile fattore di rischio per la malattia. Questa associazione è supportata dalla precedente conoscenza che l'abuso di alcol può compromettere la funzionalità epatica (EHRMANN; URBAN; DVORAN, 2019), che spaventerebbe il paziente a sviluppare complicazioni e, di conseguenza, un quadro più grave del COVID-19.

La relazione tra puerperium e COVID-19 non è così consolidata rispetto alla gravidanza (AN et al., 2020). Nel frattempo, Tutiya et al. (2020) ha segnalato due casi di formazione polmonare di microtrombi nelle donne puerperali a cui è stato diagnosticato covid-19. Inoltre, An et al. (2020) ha segnalato due casi di donne puerperali che hanno presentato disagio respiratorio e peggioramento delle condizioni dopo il parto. Queste osservazioni rafforzano la necessità di monitorare le condizioni dei pazienti dopo il parto fino a quando non vi è stabilizzazione.

Dengue e malattie pancreatiche che sono state riportate nei decessi dovuti al COVID19 ad Amapá, tuttavia, non sono stati trovati rapporti nella letteratura sull'associazione di queste malattie e sullo sviluppo di complicanze dellinfezione virale, sebbene le malattie che compromettono l'immunità del paziente tendano ad aumentare le possibilità di peggioramento delle condizioni preesistenze (MARTINS-CHAVES; GOMES GOMEZ, 2020).

\section{CONCLUSIONE}

La preessistenza di comorbilità o altre condizioni che compromettono la risposta immunitaria del corpo è un potenziale fattore aggravante della condizione infettiva generata dal nuovo coronavirus, che può portare il paziente alla morte. Nello stato di Amapá, gran parte della popolazione di pazienti a cui è stata diagnosticata la malattia e che sono morti ha avuto una storia di fattore di rischio per COVID-19. 
Le principali comorbilità riportate nei pazienti con diagnosi di malattia erano diabete, malattie cardiache croniche, malattie respiratorie croniche e ipertensione. La comorbilità con il record più alto nei casi di morte era l'ipertensione.

La notifica dei fattori di rischio nel grafico della morte che non sono stati registrati nel quadro dei pazienti diagnosticati, nonché la non specificità inerente alle condizioni dei pazienti monitorati dai meccanismi sanitari dello Stato hanno effettuato un'analisi più dettagliata sul grado di complicanza e tasso di letalità generato da una data comorbilità quando è stato associato all'infezione da coronavirus.

Per una maggiore completezza e affidabilità dei risultati di uno studio come questo, è essenziale ottenere dati sufficientemente accurati e coerenti, in modo che il passaggio dei pazienti attraverso la rete sanitaria possa essere analizzato e quindi incluso nella ricerca scientifica, rendendo possibile comprendere e affrontare l'attuale pandemia e le sue ripercussioni in Amapá.

\section{RIFERIMENTI}

AGHAGOLI, G. et al. Cardiac involvement in COVID-19 patients: Risk factors, predictors, and complications: A review. J Card Sug., v. 35, p. 1302-1305, abr. 2020.

ALMEIDA, J. S. et al. Caracterização epidemiológica dos casos de covid-19 no maranhão: uma breve análise. Revista Prevenção de Infecção e Sáude, v. 6, mai. 2020.

AL-QUTEIMAT, O. M.; AMER, A. M. The Impact of the COVID-19 Pandemic on Cancer Patients. Am J Clin Oncol., abr. 2020. DOI: 10.1097/COC.0000000000000712.

ALQAHTANI, J. S. et al. Prevalence, Severity and Mortality associated with COPD and Smoking in patients with COVID-19: A Rapid Systematic Review and Meta-Analysis. PLoS One, v. 15, n. 5, e0233147, mai. 2020.

AN, P. et al. Postpartum exacerbation of antenatal COVID-19 pneumonia in 3 women. CMAJ, v. 192, n. 22, p. 603-606, jun. 2020. 
ANGHEBEM, M. I.; REGO, F. G. M.; PICHETH, G. COVID-19 e Diabetes: a relação entre duas pandemias distintas. Revista Brasileira de Análises Clínicas. DOI: 10.21877/2448-3877.20200001. 2020.

ASKIN, L.; TANRIVERDI, O.; ASKIN, H. S. The Effect of Coronavirus Disease 2019 on Cardiovascular Diseases. Arq. Bras. Cardiol., São Paulo, v. 114, n. 5, p. 817-822, mai. 2020.

ASOKAN, I.; RABADIA, S. V.; YANG, E. H. The COVID-19 Pandemic and its Impact on the Cardio-Oncology Population. Curr Oncol Rep., v. 22, n. 6, mai. 2020.

BIANCHETTI, A. et al. Clinical presentation of COVID-19 in dementia patients. J Nutr Health Aging, mai., 2020. DOI: 10.1007/s12603-020-1389-1.

BORNSTEIN, S. R. et al. Practical recommendations for the management of diabetes in patients with COVID-19. The lancet Diabetes \& endocrinology, v. 9, n. 6, p. 546550, jun. 2020.

BRASIL. Plano de contingência DATASUS: Situação de crise provocada pelo novo coronavírus (covid-19). Versão 1.0, 2020a.

BRASIL. MINISTÉRIO DA SAÚDE. Alzheimer: o que é, causas, sintomas, tratamento, diagnóstico e prevenção. Disponível em:<https://saude.gov.br/saudede-a-z/alzheimer>. Acesso em: 31 jul. $2020 b$.

BRASIL. MINISTÉRIO DA SAÚDE. Painel de casos de doença pelo coronavírus 2019 (COVID-19) no Brasil pelo Ministério da Saúde. Versão 2.0. Disponível em:< https://covid.saude.gov.br/>. Acesso em: 10 de jul. de 2020c.

BUSSALINO, E. et al. Immunosuppressive therapy maintenance in a kidney transplant recipient SARS-CoV-2 pneumonia: a case report. Am J Transplant., abr. 2020. DOI: 10.1111/ajt.15920. 
CAPARRO-GONZALEZ, R. A. COVID-19 in PregnantWomen and Neonates: A Systematic Review of the Literature with Quality Assessment of the Studies. Rev Esp Salud Pública., v. 94, abr. 2020.

CHENG, $Y$ et al. Kidney disease is associated with in-hospital death of patients with COVID-19. Kidney Internacional, v. 97, n. 5, p. 829-838, mar. 2020.

CHINAZZI, M. et al. The effect of travel restrictions on the spread of the 2019 novel coronavirus (COVID-19) outbreak. Science, v. 368, n. 6489, p. 395-400, mar. 2020.

COSTA, I. B. S. S. et al. O Coração e a COVID-19: O que o Cardiologista Precisa Saber. Arq. Bras. Cardiol., v. 114, n. 5, p. 805-816, mai. 2020a.

COSTA, F. F. et al. Metabolic syndrome and COVID-19: An update on the associated comorbidities and proposed therapies. Diabetes \& Metabolic Syndrome: Clinical Research \& Reviews, v. 14, n. 5, p. 809-814, 2020b.

DIAS, N. L. et al. Predição da propagação do SARS-CoV-2 no Estado do Amapá, Amazônia, Brasil, por modelagem matemática. Revista Científica Multidisciplinar Núcleo do Conhecimento, vol. 06, ano 05, ed. 05, p 73-95, mai., 2020.

DRIGGIN, E. et al. Cardiovascular Considerations for Patients, Health Care Workers, and Health Systems During the COVID-19 Pandemic. J Am Coll Cardiol., v. 75, n. 18, p. 2352-2371, mai. 2020.

ENGIN, A. B.; ENGIN, E. D.; ENGIN, A. Two important controversial risk factors in SARS-CoV-2 infection: obesity and smoking. Environmental Toxicology and Pharmacology, v. 78, mai. 2020. DOI: 10.1016/j.etap.2020.103411.

ENGSTROM, E. et al. Recomendações para a organização da Atenção Primária à Saúde no SUS no enfrentamento da Covid-19. Observatório Covid-19 Fiocruz, Nota técnica. p. 13, mai. 2020. 
EHRMANN, J.; URBAN, O.; DVORAN, P. Alcohol-related liver diseases. Cent Eur J Public Health, v. 27, p. 10-14, dez. 2019.

FAN, C. et al. ACE2 Expression in Kidney and Testis May Cause Kidney and Testis Damage After 2019-nCoV Infection. MedRxiv, fev. 2020. DOI: 10.1101/2020.02.12.20022418.

FANG, L.; KARAKIULAKIS, G.; ROTH, M. Are patients with hypertension and diabetes mellitus at increased risk for COVID-19 infection?. The Lancet. Respiratory Medicine, v. 8, n. 4, mai. 2020.

FERINI-STRAMBI, L.; SALSONE, M. COVID-19 and neurological disorders: are neurodegenerative or neuroimmunological diseases more vulnerable?. J Neurol., jul. 2020. DOI: 10.1007/s00415-020-10070-8.

FERRARI, F. COVID-19: Dados Atualizados e sua Relação Com o Sistema Cardiovascular. Arq. Bras. Cardiol., v. 114, n. 5, p. 823-826, mai. 2020. DOI: 10.36660/abc.20200215.

GAUTRET, P. et al. Hydroxychloroquine and azithromycin as a treatment of COVID19: results of an open-label non-randomized clinical trial. International journal of antimicrobial agents, mar. 2020. DOI: 10.1016/j.jjantimicag.2020.105949.

GIMENEZ, V. M. M. et al. Lungs as target of COVID-19 infection: Protective common molecular mechanisms of vitamin $D$ and melatonin as a new potential synergistic treatment. Life Sci., v. 354, ago. 2020. DOI: 10.1016/j.lfs.2020.117808.

GUAN, W. et al. Clinical characteristics of coronavirus disease 2019 in China. New England journal of medicine, v. 382, n. 18, p. 1708-1720, fev. 2020.

HONG, H. et al. Clinical characteristics of novel coronavirus disease 2019 (COVID-19) in newborns, infants and children. Pediatrics and Neonatology, v. 61, n. 2, p. 131132, abr. 2020. 
HRUSAK, O. et al. Flash survey on severe acute respiratory syndrome coronavirus-2 infections in paediatric patients on anticancer treatment. European Journal of Cancer, v. 132, p. 11-16, abr. 2020.

HUANG, C.; WANG, Y.; LI, X. Características clínicas de pacientes infectados com o novo coronavírus de 2019 em Wuhan, China. Lanceta, v. 395, p. 497-506, jan. 2020.

HUSSAIN, A. Obesity and mortality of COVID-19. Meta-analysis. Obes Res Clin Pract., jul. 2020. DOI: 10.1016/j.orcp.2020.07.002.

KHALIQ, O. P.; PHOSWA, W. N. Is Pregnancy a Risk Factor of COVID-19?. European Journal of Obstetrics \& Gynecology and Reproductive Biology, 2020. DOI: 10.1016/j.ejogrb.2020.06.058.

IACOBELLIS, G. COVID-19 and diabetes: can DPP4 inhibition play a role?. Diabetes research and clinical practice, v. 162, abr. 2020. DOI: 10.1016/j.diabres.2020.108125.

LI, H. et al. Coronavirus disease 2019 (COVID-19): current status and future perspective. International journal of antimicrobial agents, v. 55, n. 5, mai. 2020.

LIU, H. et al. Why are pregnant women susceptible to COVID-19? An immunological Viewpoint. Journal of reproductive immunology, v. 139, jun. 2020.

LIMA, D. L. P. et al. COVID-19 no estado do Ceará, Brasil: comportamentos e crenças na chegada da pandemia. Ciênc. saúde coletiva, v. 25, n. 5, p. 1575-1586, mai. 2020.

LUCENA, T. M. C. et al. Mechanism of inflammatory response in associated comorbidities in COVID-19. Diabetes \& Metabolic Syndrome: Clinical Research \& Reviews, mai. 2020. DOI: 10.1016/j.dsx.2020.05.025.

MA, R. C. W.; HOLT, R. I. G. COVID-19 and diabetes. Diabetic Medicine, abr. 2020. DOI: $10.1111 / \mathrm{dme} .14300$. 
MARTINS-CHAVES, R. R.; GOMES, C. C.; GOMEZ, R. S. Immunocompromised patients and coronavirus disease 2019: a review and recommendations for dental health care. Braz. res oral., São Paulo, v. 34, e048, mai. 2020.

MENDONÇA, F. D. Região Norte do Brasil e a pandemia de COVID-19: análise socioeconômica e epidemiológica. Journal Health NPEPS, v. 5, n. 1, 2020.

MOITINHO, M. S. et al. Lesão renal aguda pelo vírus SARS-COV-2 em pacientes com COVID-19: revisão integrativa. Rev. Bras. Enferm., Brasília, v. 73, n. 2, jul. 2020.

OLIVEIRA, A. C.; LUCAS, T. C.; IQUIAPAZA, R. A. What has the COVID-19 pandemic taught us about adopting preventive measures?. Texto \& Contexto Enfermagem, Florianópolis, v. 29, mai. 2020. DOI: 10.1590/1980-265x-tce-2020-0106.

OLIVEIRA, W. K. et al. Como o Brasil pode deter a COVID-19. Epidemiol. Serv. Saude, Brasília, v. 9, n. 2, p. 1-8, abr. 2020.

OMS. ORGANIZAÇÃO MUDIAL DA SAÚDE. Painel da Doença de Coronavírus da OMS (COVID-19). Disponível em:< https://covid19.who.int/>. Acesso em: 10 de jul. de 2020.

OYELADE, T.; ALQAHTANI, J.; CANCIANI, G. Prognosis of COVID-19 in Patients with Liver and Kidney Diseases: An Early Systematic Review and Meta-Analysis. Trop. Med. Infect. Dis., v. 5, n. 80, mai. 2020.

PRANATA, R. et al. Impact of cerebrovascular and cardiovascular diseases on mortality and severity of COVID-19-systematic review, meta-analysis, and metaregression. J Stroke Cerebrovasc Dis., v. 29, n. 8,: mai. 2020.

PIRES, L. N.; CARVALHO, L.; XAVIER, L. L. COVID-19 e desigualdade: a distribuição dos fatores de risco no Brasil. Research Gate, abr. 2020. DOI: 10.13140/RG.2.2.27014.73282.

RAJKUMAR, R. P. Ayurveda and COVID-19: Where psychoneuroimmunology and the meaning response meet. Brain, behavior and immunity, v. 87, p. 8-9, jul. 2020. 
RENU, K.; PRASANNA, P. L.; GOPALAKRISHNAN, A. V. Coronaviruses pathogenesis, comorbidities and multi-organ damage - A review. Life sci., v. 255, mai. 2020. DOI: $10.1016 / j . I f s .2020 .117839$.

RODRÍGUEZ-COLA, M. et al. Clinical features of coronavirus disease 2019 (COVID19 ) in a cohort of patients with disability due to spinal cord injury. Spinal Cord Series and Cases, v. 6, n. 39, mai. 2020.

RONCON, L. et al. Diabetic patients with COVID-19 infection are at higher risk of ICU admission and poor short-term outcome. Journal of Clinical Virology, v. 127, jun. 2020. DOI: 10.1016 / j.jcv.2020.104354.

RUBIO-PÉREZ, I. et al. COVID-19: Conceptos clave para el cirujano. Cirugía Española, v. 98, n. 6, p. 310-319, jun./jul. 2020.

SIA, S. F. et al. Pathogenesis and transmission of SARS-CoV-2 in golden hamsters. Nature, mai. 2020. DOI: 10.1038/s41586-020-2342-5.

SILVA, A.W.C. et al. Caracterização clínica e epidemiologia de 1560 casos de COVID19 em Macapá/AP, extremo norte do Brasil. Research, Society and Development, v. 9, n.8, e150985499, jun. 2020.

STEFAN. N. et al. Obesity and impaired metabolic health in patients with COVID-19. Nat Rev Endocrinol., abr. 2020. DOI: 10.1038/s41574-020-0364-6.

TAY, M. Z. et al. The trinity of COVID-19: immunity, inflammation and intervention. Nat Rev Immunol, v. 20, p. 363-374, abr. 2020. DOI: 10.1038/s41577-020-0311-8.

TADIC, M. et al. COVID-19 and arterial hypertension: Hypothesis or evidence? J Clin Hypertens (Greenwich), jul. 2020. DOI:10.1111/jch.13925.

TRIPPELA, G. et al. COVID-19 in PregnantWomen and Neonates: A Systematic Review of the Literature with Quality Assessment of the Studies. Pathogens, v. 9, n. 6, jun. 2020. 
TROYER, E. A.; KOHN, J. N.; HONG, S. Are we facing a crashing wave of neuropsychiatric sequelae of COVID-19? Neuropsychiatric symptoms and potential immunologic mechanisms. Brain, behavior and immunity, v. 87, p. 34-39, jul. 2020.

TUTIYA, C. T. et al. Possible formation of pulmonary microthrombi in the early puerperium of pregnant women critically ill with COVID-19: Two case reports. Case Rep Womens Health, v. 27, e00237, jun. 2020.

VARDAVAS, C. I.; NIKITARA, K. COVID-19 and smoking: A systematic review of the evidence. Tob Induc Dis., v. 18, mar. 2020. DOI: 10.18332/tid/119324.

WANG, D. et al. Clinical Characteristics of 138 Hospitalized Patients With 2019 Novel Coronavirus-Infected Pneumonia in Wuhan, China. Journal of American Medical Asssociation, v. 323, n. 11, p. 1061-1069, fev. 2020.

YANG, J. et al. Prevalence of comorbidities and its effects in patients infected with SARS-CoV-2: a systematic review and meta-analysis. Int $\mathbf{J}$ Infect Dis., v. 94, p. 9195, mai. 2020.

YAO, H.; CHEN, J. H.; XU, Y. F. Patients with mental health disorders in the COVID19 epidemic. Lancet Psychiatry, v. 7, n. 4, mar. 2020.

ZHAI, P. et al. The epidemiology, diagnosis and treatment of COVID-19. International journal of antimicrobial agents, v. 55, n. 5, mai. 2020.

ZHANG, L. et al. Clinical characteristics of COVID-19-infected cancer patients: a retrospective case study in three hospitals within Wuhan, China. Ann Oncol., v. 31, n. 7, p 894-901, jul. 2020.

Inviato: Giugno 2021.

Approvato: Giugno 2021. 